TRANSACTIONS OF THE

AMERICAN MATHEMATICAL SOCIETY

Volume 185. November 1973

\title{
REPRESENTATIONS OF JORDAN TRIPLES
}

\author{
BY \\ OTTMAR LOOS
}

\begin{abstract}
Some standard results on representations of quadratic Jordan algebras are extended to Jordan triples. It is shown that the universal envelope of a finite-dimensional Jordan triple is finite-dimensional, and that it is nilpotent if the Jordan triple is radical. A permanence principle and a duality principle are proved which are useful in deriving identities.
\end{abstract}

Introduction. A Jordan triple is a module $V$ over a commutative ring $k$ together with a composition $(x, y) \mapsto P(x) y$ which is quadratic in $x$ and linear in $y$ and satisfies certain identities (see (1)-(3) below). A typical example is the space of $p \times q$-matrices over $k$ with $P(x) y=x\left({ }^{t} y\right) x$. If $J$ is a quadratic Jordan algebra with quadratic operators $U_{x}$ then $J$ is also a Jordan triple with $P(x) y=U_{x} y$. Thus Jordan triples are a natural generalization of quadratic Jordan algebras. For a systematic theory of Jordan triples see [2] and [5].

In this note, we extend to Jordan triples certain standard results from the representation theory of quadratic Jordan algebras (see [4]). Our main results concern the case where $V$ is finite-dimensional over a field $k$. Then the universal envelope of $V$ is also finite-dimensional (Theorem 2.4), and it is nilpotent in case $V$ is radical (Theorem 3.3). The latter result is due to C.T. Anderson in case char $k \neq 2$. We also prove a permanence principle and a duality principle which are useful in deriving identities.

In [6], K. Yamaguti also defines representations of Jordan triple systems. However, his concept of Jordan triple system is different from ours (the Jordan triple systems of type II considered in [6] are a generalization of our Jordan triples).

\section{Representations.}

1.1. Jordan triples. Let $k$ be a commutative ring with unit and let $V$ and $W$ be unital $k$-modules. A map $P: V \rightarrow W$ is called quadratic if $P(\alpha x)=\alpha^{2} P(x)$ for all $\alpha \in k, x \in V$, and if $P(x, y)=P(x+y)-P(x)-P(y)$ is bilinear in $x$ and $y$. If $R$ is any commutative associative $k$-algebra then there is a unique quadratic map $P_{R}: V \otimes_{k} R \rightarrow W \otimes_{k} R$ of $R$-modules making the diagram

Received by the editors May 12, 1972.

AMS (MOS) subject classifications (1970). Primary 17C15, 17C25, 17 C99.

Key words and phrases. Jordan triple, Jordan algebra, representation, universal envelope, nilpotence.

Copyright O 1974, American Mathematical Society 


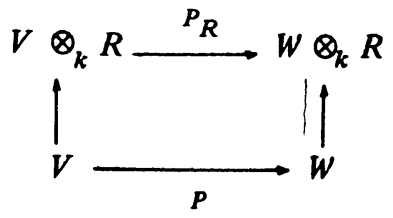

commutative (see [1]). In case $W=$ End $_{k} V$, we denote the composition $V \otimes_{k} R$ $\rightarrow\left(\operatorname{End}_{k} V\right) \otimes_{k} R \rightarrow \operatorname{End}_{R}\left(V \otimes_{k} R\right)$ also by $P_{R}$.

Let now $P: V \rightarrow$ End $_{k} V$ be a quadratic map. We set

$$
\{x y z\}=L(x, y) z=P(x, z) y .
$$

Then $(x, y, z) \mapsto\{x y z\}$ is a $k$-trilinear map from $V \times V \times V$ into $V$ such that $\{x y z\}=\{z y x\}$ and $\{x y x\}=2 P(x) y$. The pair $(V, P)$ is called a Jordan triple if the identities

$$
\begin{aligned}
L(x, y) P(x) & =P(x) L(y, x)=P(P(x) y, x), \\
L(P(x) y, y) & =L(x, P(y) x), \\
P(P(x) y) & =P(x) P(y) P(x)
\end{aligned}
$$

hold in $V$ and in all scalar extensions $\left(V_{R}, P_{R}\right)$ of $(V, P)$ (equivalently, if all linearizations of (1)-(3) hold in $V$ ).

A $k$-linear map $f: V \rightarrow W$ of Jordan triples is called a homomorphism if $f(P(x) y)=P(f(x)) f(y)$ for all $x, y \in V$. An ideal of $V$ is a $k$-submodule $I$ satisfying $P(I) V+P(V) I+\{V V I\} \subset I$. For the general theory of Jordan triples see [2], [5].

1.2. Identities. By linearizing (1) we obtain

$$
\begin{aligned}
L(x, y) P(x, z)+L(z, y) P(x) & =P(x, z) L(y, x)+P(x) L(y, z) \\
& =P(\{x y z\}, x)+P(P(x) y, z) .
\end{aligned}
$$

We apply this to an element $u \in V$, regard it as a function of $z$ and change $u$ to z. Then we have

$$
L(x, y) L(x, z)+L(P(x) z, y)=L(x,\{y x z\})+P(x) P(y, z) .
$$

We linearize (2) with respect to $x$ and $y$ and obtain

$$
\begin{aligned}
L(\{x y z\}, y) & =L(z, P(y) x)+L(x, P(y) z), \\
L(x,\{y x z\}) & =L(P(x) y, z)+L(P(x) z, y) .
\end{aligned}
$$

Again we apply this to an element of $V$ and regard it as a function of $z$ and obtain, after a change of notation,

$$
\begin{aligned}
L(z, y) L(x, y) & =P(x, z) P(y)+L(z, P(y) x), \\
P(x, z) L(y, x) & =P(P(x) y, z)+L(z, y) P(x) .
\end{aligned}
$$


Subtract (9) from (4) to obtain

$$
P(x) L(y, z)+L(z, y) P(x)=P(x,\{x y z\}) .
$$

Addition of (5) and (7) gives

$$
L(x, y) L(x, z)=L(P(x) y, z)+P(x) P(y, z) .
$$

1.3. Definition. Let $V$ be a Jordan triple over $k$, and let $A$ be a unital associative $k$-algebra. A representation of $V$ in $A$ is a pair $(l, p)$ of maps where $l: V \times V \rightarrow A$ is bilinear and $p: V \rightarrow A$ is quadratic, such that the following identities hold in all scalar extensions.

$$
\begin{aligned}
l(x, y) p(x)=p(x) l(y, x) & =p(x, P(x) y), \\
p(x) l(y, z)+l(z, y) p(x) & =p(x,\{x y z\}), \\
l(x, y) l(x, z) & =l(P(x) y, z)+p(x) p(y, z), \\
l(z, x) l(y, x) & =l(z, P(x) y)+p(y, z) p(x), \\
p(P(x) y) & =p(x) p(y) p(x) .
\end{aligned}
$$

If $A$ has an involution $a \mapsto a^{*}$ such that $l(x, y)^{*}=l(y, x)$ and $p(x)^{*}=p(x)$ for all $x, y \in V$ then $(l, p)$ is called a ${ }^{*}$-representation. In this case, (15) is a consequence of (14).

Example. (a) The regular representation $(L, P)$ of $V$ in $\operatorname{End}_{k} V$.

(b) The regular *-representation of $V$ in $E=\operatorname{End}_{k} V \times\left(\operatorname{End}_{k} V\right)^{\text {op }}$ given by

$$
l(x, y)=(L(x, y), L(y, x)) \text { and } p(x)=(P(x), P(x)) .
$$

The interchange $(f, g) \mapsto(g, f)$ is an involution of $E$ making $(l, p)$ a *representation.

1.4. Lemma. If $(l, p)$ is a representation of $V$ in $A$ then the following formulas hold.

$$
\begin{aligned}
l(P(x) y, y) & =l(x, P(y) x), \\
p(x, z) l(y, x) & =l(z, y) p(x)+p(P(x) y, z), \\
l(x, y) p(x, z) & =p(x) l(y, z)+p(P(x) y, z) .
\end{aligned}
$$

Proof. (17) follows by setting $y=z$ in (14) and (15) and subtracting. We linearize (12):

$$
\begin{aligned}
l(z, y) p(x)+l(x, y) p(x, z) & =p(x) l(y, z)+p(x, z) l(y, x) \\
& =p(x,\{x y z\})+p(z, P(x) y),
\end{aligned}
$$

subtract (13) and obtain (18) and (19). 
1.5. Split null extensions. If $M$ is a $k$-module and $(l, p)$ is a representation of $V$ in $\operatorname{End}_{k} M$ then we say $M$ is a $V$-module. As in the case of quadratic Jordan algebras (see [4]) we have

Proposition. $V \oplus M$ becomes a Jordan triple with

$$
P(x \oplus m)(y \oplus n)=P(x) y \oplus[p(x) n+l(x, y) m]
$$

$(x, y \in V, m, n \in M)$, the split null extension of $V$ by $M$.

Proof. If we use the fact that any product in $V \oplus M$ containing more than one element from $M$ is zero, as well as the identities (12)-(19), the verification of (1) in $V \oplus M$ amounts to

$$
\begin{aligned}
p(x) p(y, z)+l(x,\{y x z\}) & =l(x, y) l(x, z)+l(P(x) z, y) \\
& =l(P(x) y, z)+l(x, z) l(x, y) .
\end{aligned}
$$

But this is an easy consequence of (14) and (17). Similarly, (2) follows without difficulty from (15), (17), and (18), and (3) comes down to showing

$$
l(P(x) y, z) p(x)=p(x) l(y, P(x) z)
$$

and

$$
p(x) p(y) l(x, z)+l(x, P(y) P(x) z)=l(P(x) y, z) l(x, y) .
$$

By (13), (12), and (17) we have

$$
\begin{aligned}
p(x) l(y, P(x) z) & +l(P(x) z, y) p(x)=p(x,\{x, y, P(x) z\}) \\
= & p(x, P(x)\{y x z\})=l(x,\{y x z\}) p(x) \\
= & l(P(x) y, z) p(x)+l(P(x) z, y) p(x)
\end{aligned}
$$

which proves (20). For (21), we use (19) and (15) and get

$$
\begin{aligned}
p(x) p(y) l(x, z)+l(x, P(y) P(x) z) & \\
= & p(x) l(y, x) p(y, z) \\
& -p(x) p(P(y) x, z)+l(x, y) l(P(x) z, y)-p(x) l(z, x) p(y) \\
= & l(x, y) l(x, z) l(x, y)-p(x) p(P(y) x, z)-p(x) l(z, x) p(y)
\end{aligned}
$$

(by (12) and (14))

$$
=l(P(x) y, z) l(x, y)+p(x)[p(y, z) l(x, y)-p(P(y) x, z)-l(z, x) p(y)]
$$

$$
=l(P(x) y, z) l(x, y) \quad(\text { by }(18)) .
$$


We remark that the discussion in [4] concerning the cohomology of quadratic Jordan algebras can be carried over word for word to the Jordan triple case, so we omit the details.

1.6. Let $\left(J T_{k}\right)$ denote the class of all Jordan triples over $k$. As in [4], we obtain from 1.5 the following

Permanence principle. If $F$ is any identity in the $L(x, y)$ 's and $P(z)$ 's which is valid for the regular representation of all $V \in\left(J T_{k}\right)$ then the identity obtained from $F$ by replacing $L, P$ by $l, p$ is valid for all representations of all $V \in\left(J T_{k}\right)$.

Indeed, to prove $F$ for a representation $(l, p)$ of $V$ in $A$, consider $A$ as a $V$ module by composing $(l, p)$ with the left regular representation of $A$. Then $F$ is valid for the regular representation of the split null extension $V \oplus A$, and by restricting to $A$ and applying $F$ to the unit element of $A$ the assertion follows.

Another useful device in deriving identities is the

Duality principle. If $F$ is any identity in $l(x, y)$ 's and $p(z)$ 's which is valid for every representation of all $V \in\left(J T_{k}\right)$ then its dual $F^{*}$, obtained by replacing $l(x, y)$ by $l(y, x)$ and reversing the order of the $l(x, y)$ 's and $p(z)$ 's, is also valid for every representation.

Indeed, $F$ holds in particular for the regular *-representation of $V$ in $E=\operatorname{End}_{k} V \times\left(\operatorname{End}_{k} V\right)^{\mathrm{op}}$. Applying the involution of $E$ and projecting onto the first factor End $_{k} V$ of $E$, we see that $F^{*}$ holds for all regular representations. By the permanence principle, $F^{*}$ holds for all representations.

1.7. Homotopes. Let $a \in V$. With the operations

$$
U_{x} y=P(x) P(a) y, \quad x^{2}=P(x) a,
$$

the $k$-module $V$ becomes a quadratic Jordan algebra $V_{a}$, the homotope of $V$ with respect to $a$ (cf. [5]). Let $\hat{V}_{a}=k .1 \oplus V_{a}$ be the unital quadratic Jordan algebra obtained from $V_{a}$ by adjoining a unit element. Recall that a unital quadratic representation of a unital quadratic Jordan algebra $J$ in a unital associative algebra $A$ is a quadratic map $\mu: J \rightarrow A$ satisfying the following identities in all scalar extensions (cf. [4]):

$$
\begin{aligned}
\mu(1) & =1, \\
\mu\left(U_{x} y\right) & =\mu(x) \mu(y) \mu(x), \\
\nu(x, y) \mu(x) & =\mu(x) \nu(y, x)=\mu\left(U_{x} y, x\right),
\end{aligned}
$$

where $\nu(x, y)=\mu(x, 1) \mu(y, 1)-\mu(x, y)$.

Proposition. Let $(l, p)$ be a representation of the Jordan triple $V$ in $A$. Then for every $a \in V$,

$$
\mu(\alpha .1+x)=\alpha^{2} .1+\alpha l(x, a)+p(x) p(a)
$$

defines a unital quadratic representation of $\hat{V}_{a}$ in $A$. 
Proof. Let $\bar{\mu}(\alpha .1+x)=U(\alpha .1+x) \mid V_{a}=\alpha^{2} \operatorname{Id}_{V}+\alpha L(x, a)+P(x) P(a)$. Since $V_{a}$ is an ideal of $\nabla_{a}$, this is a unital quadratic representation of $\nabla_{a}$, and the validity of (23) and (24) for $\bar{\mu}$ is equivalent to certain identities in L's and P's. By the permanence principle, the same identities hold with $L, P$ replaced by $l, p$, i.e., for $\mu$. Hence $\mu$ satisfies (23) and (24). Since it is obviously quadratic and $\mu(1)=1$, the proposition follows.

Recall that a pair $(x, a) \in V \times V$ is called quasi-invertible if $1-x$ is invertible in $\hat{V}_{a}$ (cf. [5]).

Corollary. If $(x, a)$ is quasi-invertible then

$$
b(x, a)=1-l(x, a)+p(x) p(a)
$$

is invertible in $A$.

Indeed, $b(x, a)=\mu(1-x)$, and $\mu$ maps invertible elements of $\hat{\nabla}_{a}$ into invertible elements of $A$.

1.8. Definition. A $\lambda$-representation of $V$ in a unital associative algebra $A$ is a bilinear map $l: V \times V \rightarrow A$ such that the identities

$$
\begin{gathered}
l(P(x) y, y)=l(x, P(y) x) \\
l(x, y) l(u, v)-l(u, v) l(x, y)=l(\{x y u\}, v)-l(u,\{y x v\}), \\
l(x, y)^{4}=l(x, y)^{2} l(P(x) y, y)+l(P(x) y, y) l(x, y)^{2} \\
+l(P(x) y, y)^{2}-l(P(P(x) y) y, y)-l(x, P(P(y) x) x)
\end{gathered}
$$

hold in all scalar extensions.

A $\pi$-representation of $V$ in $A$ is a quadratic map $p: V \rightarrow A$ such that the identities

$$
\begin{aligned}
& p(x+y)=p(x)+p(y), \\
& p(P(x) y)=p(x) p(y) p(x)
\end{aligned}
$$

hold in all scalar extensions.

Lemma. (a) If $(l, p)$ is a representation of $V$ then $l$ is a $\lambda$-representation.

(b) If $p$ is a $\pi$-representation then $2 p(x)=0$ for all $x \in V$ and

$$
p(\{x y z\})=p(x) p(y) p(z)+p(z) p(y) p(x) .
$$

Proof. (a) (25) follows from the corresponding identity for the regular representation (see [5]) by the permanence principle. If we set $y=z$ in (14) we get $l(x, y)^{2}-l(P(x) y, y)=2 p(x) p(y)$. By squaring this and using (16) and (17) we get (26).

(b) By (27), $2 p(x)=p(2 x)=4 p(x)$, and (28) follows from 


$$
\begin{aligned}
p(\{x y z\}) & =p(P(x+z) y-P(x) y-P(z) y) \\
& =p(P(x+z) y)+p(P(x) y)+p(P(z) y) \\
& =p(x+z) p(y) p(x+z)+p(x) p(y) p(x)+p(z) p(y) p(z) \\
& =p(x) p(y) p(z)+p(z) p(y) p(x)+2 p(x) p(y) p(x)+2 p(z) p(y) p(z) .
\end{aligned}
$$

\section{Universal envelopes.}

2.1. We first construct a universal object for quadratic maps. Let $\boldsymbol{V}$ be a $\boldsymbol{k}$ module, let $q: V \rightarrow X$ be a bijection of $V$ onto a set $X$, and let $F$ be the free $k$ module generated by $X$. We set $q(x, y)=q(x+y)-q(x)-q(y)$, and let $R$ be the submodule of $F$ generated by

$$
\begin{aligned}
& q(\alpha x)-\alpha^{2} q(x), \quad q(\alpha x, y)-\alpha q(x, y), \\
& q(x+y, z)-q(x, z)-q(y, z),
\end{aligned}
$$

where $\alpha \in k, x, y, z \in V$. We set $V^{I I}=F / R$ and denote the image of $q(x)$ under the canonical map by $x^{\text {II }}$. We also set $\langle x, y\rangle=(x+y)^{\mathrm{II}}-x^{\mathrm{II}}-y^{\mathrm{II}}$. Then $x \mapsto x^{\mathrm{Il}}$ is a quadratic map, $V^{\mathrm{Il}}$ is generated by $\left\{x^{\mathrm{II}}: x \in V\right\}$, and for any quadratic map $Q: V \rightarrow W$ there is a unique linear map $f: V^{\text {II }} \rightarrow W$ such that $Q(x)=f\left(x^{I I}\right)$. Also it is easily seen that $V^{I I}$ is functorial in $V$ and compatible with extensions of the ring of scalars.

2.2. The universal envelope. Let $V$ be a Jordan triple over $k$, let $W=V^{\text {II }}$ $\oplus\left(V \otimes_{k} V\right)$, and let $T(W)$ be the tensor algebra over $W$. The product of two elements $u, v \in \mathbf{T}(W)$ is denoted by $u \cdot v$.

Let $\mathbf{J}$ be the ideal of $\mathbf{T}(W)$ generated by the elements

$$
\begin{aligned}
& (x \otimes y) \cdot x^{\mathrm{II}}-x^{\mathrm{II}} \cdot(y \otimes x), \quad(x \otimes y) \cdot x^{\mathrm{II}}-\langle x, P(x) y\rangle, \\
& x^{\mathrm{II}} \cdot(y \otimes z)+(z \otimes y) \cdot x^{\mathrm{II}}-\langle x,\{x y z\}\rangle, \\
& (x \otimes y) \cdot(x \otimes z)-P(x) y \otimes z-x^{\mathrm{II}} \cdot\langle y, z\rangle, \\
& (z \otimes x) \cdot(y \otimes x)-z \otimes P(x) y-\langle y, z\rangle \cdot x^{\mathrm{II}}, \\
& (P(x) y)^{\mathrm{II}}-x^{\mathrm{II}} \cdot y^{\mathrm{II}} \cdot x^{\mathrm{II}},
\end{aligned}
$$

corresponding to (12)-(16). The universal envelope of $V$ is $\mathbf{U}(V)=\mathbf{T}(W) / \mathrm{J}$. We define $\tilde{l}: V \times V \rightarrow \mathrm{U}(V)$ by $\tilde{l}(x, y)=x \otimes y+\mathbf{J}$ and $\tilde{p}: V \rightarrow \mathbf{U}(V)$ by $\tilde{p}(x)$ $=x^{\mathrm{II}}+\mathbf{J}$.

Proposition. (a) There is an involution * of $\mathrm{U}(V)$ such that $(\tilde{l}, \tilde{p})$ is $a^{*}$. representation of $V$. For any representation $(l, p)$ of $V$ in $A$ there is a unique homomorphism $f: \mathrm{U}(V) \rightarrow A$ of unital associative algebras such that $p=f \circ \tilde{p}$ and $l=f \circ \tilde{l}$. If $(l, p)$ is $a^{*}$-representation then $f$ commutes with the involutions of $\mathrm{U}(V)$ and $A$. 
(b) There is an augmentation $\varepsilon: \mathrm{U}(V) \rightarrow k$ so that $\mathbf{U}(V)=k .1 \oplus \mathbf{U}^{\circ}(V)$ where $\mathbf{U}^{\circ}(V)=\operatorname{Ker} \varepsilon$ is the augmentation ideal. Also, $\mathbf{U}(V)$ is functorial in $V$ and is compatible with scalar extensions.

(c) If $I$ is an ideal of $V$ and $\tilde{\mathbf{I}}$ is the ideal of $\mathrm{U}(V)$ generated by $\tilde{l}(I, V), \tilde{l}(V, I)$, $\tilde{p}(I, V)$, and $\tilde{p}(I)$ then $\mathbf{U}(V / I) \cong \mathbf{U}(V) / \tilde{\mathbf{I}}$.

The proof of this proposition follows established lines and is therefore omitted. Let us just indicate how the involution ${ }^{*}$ of $\mathbf{U}(V)$ is defined. The $k$-module $W=V^{\mathrm{II}} \oplus(V \otimes V)$ possesses an endomorphism of period 2 given by $x^{\mathrm{II}} \mapsto x^{\mathrm{II}}$ and $x \otimes y \mapsto y \otimes x$. By the universal property of the tensor algebra, this endomorphism extends to an involution of $\mathbf{T}(W)$ leaving $\mathbf{J}$ invariant, and thus induces an involution * of $\mathbf{U}(V)$ with the desired properties.

2.3. Similarly as in 2.2 , we define the universal $\lambda$-envelope $U_{\lambda}(V)=$ $\mathbf{T}(V \otimes V) / \mathbf{J}_{\lambda}$ where $\mathbf{J}_{\lambda}$ is the ideal of $\mathbf{T}(V \otimes V)$ generated by the elements

$$
\begin{aligned}
& P(x) y \otimes y-x \otimes P(y) x, \\
& (x \otimes y) \cdot(u \otimes v)-(u \otimes v) \cdot(x \otimes y)-\{x y u\} \otimes v+u \otimes\{y x v\}, \\
& (x \otimes y)^{4}-(x \otimes y)^{2} \cdot(P(x) y \otimes y)-(P(x) y \otimes y) \cdot(x \otimes y)^{2} \\
& \quad-(P(x) y \otimes y)^{2}+P(P(x) y) y \otimes y+x \otimes P(P(y) x) x,
\end{aligned}
$$

corresponding to (17), (25), and (26), and the universal $\pi$-envelope $U_{\pi}(V)$ $=\mathbf{T}\left(V^{\mathrm{II}}\right) / \mathrm{J}_{\pi}$ where $\mathrm{J}_{\pi}$ is the ideal of $\mathbf{T}\left(V^{\mathrm{II}}\right)$ generated by the elements $\langle x, y\rangle$ $=(x+y)^{\mathrm{II}}-x^{\mathrm{II}}-y^{\mathrm{II}}$ and $(p(x) y)^{\mathrm{II}}-x^{\mathrm{II}} \cdot y^{\mathrm{II}} \cdot x^{\mathrm{II}}$. Define $\bar{l}: V \times V \rightarrow \mathrm{U}_{\lambda}(V)$ by $\bar{l}(x, y)=x \otimes y+J_{\lambda}$ and $\bar{p}: V \rightarrow \mathbf{U}_{\pi}(V)$ by $\bar{p}(x)=x^{I I}+J_{\pi}$.

Proposition. (a) $\bar{l}$ (resp. $\bar{p}$ ) is a $\lambda$-representation (resp. a $\pi$-representation) of $V$ in $\mathbf{U}_{\lambda}(V)$ (resp. in $\mathbf{U}_{\pi}(V)$ ), and any $\lambda_{-}$(resp. $\pi^{-}$) representation may be factored via $\mathbf{U}_{\lambda}(V)\left(\right.$ resp. $\left.\mathbf{U}_{\pi}(V)\right)$.

(b) $\mathbf{U}_{\lambda}(V)$ and $\mathbf{U}_{\eta}(V)$ are functorial in $V$ and compatible with scalar extensions. There are augmentations $\mathrm{U}_{\lambda}(V) \rightarrow k$ and $\mathrm{U}_{\pi}(V) \rightarrow k$ so that $\mathrm{U}_{\lambda}(V)=k .1$ $\oplus \mathbf{U}_{\lambda}^{\circ}(V)$ and $\mathbf{U}_{\sharp}(V)=k .1 \oplus \mathbf{U}_{\sharp}^{\circ}(V)$.

(c) If $I$ is an ideal of $V$ and if $\mathbf{I}_{\lambda}$ (resp. $\left.\mathbf{I}_{n}\right)$ is the ideal of $\mathbf{U}_{\lambda}(V)$ (resp. $\mathbf{U}_{\pi}(V)$ ) generated by $\bar{l}(I, V)$ and $\bar{l}(V, I)($ resp. $\bar{p}(I))$ then $\mathbf{U}_{\lambda}(V / I) \cong \mathbf{U}_{\lambda}(V) / \mathbf{I}_{\lambda}$ (resp. $\left.\mathbf{U}_{\pi}(V / I) \cong \mathbf{U}_{\pi}(V) / \mathbf{I}_{\pi}\right)$.

(d) $\mathrm{U}_{\lambda}(V)$ and $\mathrm{U}_{\pi}(V)$ possess involutions * such that $\bar{l}(x, y)^{*}=\bar{l}(y, x)$ and $\bar{p}(x)^{*}=\bar{p}(x)$. Also, $\mathbf{U}_{\pi}(V)=\mathbf{U}_{\pi}^{+}(V) \oplus \mathbf{U}_{\pi}^{-}(V)$ is $\mathbf{Z}_{2}$-graded, the gradation is invariant under ${ }^{*}$, and $\bar{p}(x) \in \mathrm{U}_{\pi}^{-}(V)$ for $x \in V$.

The proof of (a), (b), and (c) is again straightforward. To prove (d), let * denote the involutions of $\mathbf{T}(V \otimes V)$ (resp. $\mathbf{T}\left(V^{\mathrm{II}}\right)$ ) induced by $x \otimes y \mapsto y \otimes x$ (resp. the identity on $V^{\text {II }}$ ). Then $J_{\lambda}$ and $J_{n}$ are invariant under ${ }^{*}$, and the statement about the involutions follows. Finally, $\mathbf{J}_{\boldsymbol{q}}$ is generated by elements of odd degree (with 
respect to the natural gradation of $\left.\mathrm{T}\left(V^{\mathrm{II}}\right)\right)$, and the gradation of $\mathrm{T}\left(V^{\mathrm{II}}\right)$ is invariant under *.

2.4. Theorem. If $k$ is a field and $V$ is finite-dimensional over $k$ then so are $U(V)$, $\mathbf{U}_{\lambda}(V)$, and $\mathbf{U}_{\pi}(V)$.

Proof. Let $x_{1}, \ldots, x_{n}$ be a basis of $V$. First we show that $U_{\lambda}(V)$ is finitedimensional. As an algebra, $U_{\lambda}(V)$ is generated by 1 and $\left\{\bar{l}\left(x_{i}, x_{j}\right): i, j=1, \ldots\right.$, $n\}$. We number the $\bar{l}\left(x_{i}, x_{j}\right)$ consecutively: $y_{1}=\bar{l}\left(x_{1}, x_{1}\right), y_{2}=\bar{l}\left(x_{1}, x_{2}\right), \ldots, y_{n^{2}}$ $=\bar{l}\left(x_{n}, x_{n}\right)$.

Lemma 1. $\mathbf{U}_{\lambda}(V)$ is spanned by the monomials $y_{i_{1}} \cdots y_{i_{1}}$ where $0 \leq r \leq 3 n^{2}$ and $i_{1} \leq i_{2} \leq \cdots \leq i_{\text {r. }}$

Proof. Let $\mathbf{X}_{r}$ be the subspace of $\mathbf{U}_{\lambda}(V)$ spanned by the monomials $y_{i_{1}} \cdots y_{i_{\text {}}}$ where $s \leq r$. Clearly, $\mathbf{X}_{0}=k .1, \mathbf{X}_{r} \subset \mathbf{X}_{r+1}$, and $\mathbf{X}_{r} \cdot \mathbf{X}_{s} \subset \mathbf{X}_{r+s}$. We claim that $\mathbf{X}_{r}=\mathbf{X}_{r-1}$ if $r>3 n^{2}$. Indeed, because of (25) we have

$$
y_{i} y_{j} \equiv y_{j} y_{i} \quad \bmod \mathbf{x}_{1} \text {. }
$$

In a monomial $y_{i} \cdots y_{i}$, where $r>3 n^{2}$, at least one of the $y_{i}$, say $y_{1}$, occurs at least 4 times. By (29), we get $y_{i_{1}} \cdots y_{i_{r}} \equiv y_{1}^{4} y_{j_{1}} \cdots y_{j_{r-4}} \bmod \mathbf{X}_{r-1}$, and (26) shows $y_{1}^{4} \in \mathbf{X}_{3}$. This proves our assertion. Since $\mathbf{U}_{\lambda}(V)$ is the union of the $\mathbf{X}_{r}$, we have $\mathbf{U}_{\lambda}(V)=\mathbf{X}_{3 n^{2}}$. Finally, the ordered monomials suffice because of (29).

Lemma 2. The subalgebra $\mathrm{U}^{\prime}$ of $\mathrm{U}(V)$ generated by $\tilde{l}(V, V)$ and $\tilde{p}(V, V)$ is a finite-dimensional ideal of $\mathbf{U}(V)$.

Proof. Let $\mathrm{L}$ be the subalgebra of $\mathrm{U}(V)$ generated by $\tilde{l}(V, V)$. Since $\tilde{l}: V \times V$ $\rightarrow \mathbf{U}(V)$ is a $\lambda$-representation, $\mathbf{L}$ is a homomorphic image of $\mathbf{U}_{\lambda}^{\circ}(V)$ and therefore finite-dimensional by Lemma 1 . Let $P$ be the subalgebra of $U(V)$ generated by $\tilde{p}(V, V)$, and let $\mathbf{P}_{+}$be the subalgebra of $\mathbf{P}$ generated by $\{\tilde{p}(u, v) \tilde{p}(x, y): u, v, x, y \in V\}$. From (14) we obtain by linearizing

$$
\tilde{p}(u, v) \tilde{p}(x, y)=\tilde{l}(v, y) \tilde{l}(u, x)+\tilde{l}(u, y) \tilde{l}(v, x)-\tilde{l}(\{u y v\}, x)
$$

which implies $\mathbf{P}_{+} \subset \mathbf{L}$. Also $\mathbf{P}=\mathbf{P}_{+}+\tilde{p}(V, V)+\mathbf{P}_{+} \tilde{p}(V, V)$ shows that $\mathbf{P}$ is finite-dimensional. From (13) we get by linearizing

$$
\tilde{l}(z, y) \tilde{p}(x, w)=\tilde{p}(x,\{w y z\})+\tilde{p}(w,\{x y z\})-\tilde{p}(x, w) \tilde{l}(y, z)
$$

which implies by induction

$$
\mathbf{L P} \subset \mathbf{P L}+\mathbf{P} \text {. }
$$

This shows that $\mathbf{U}^{\prime}=\mathbf{L}+\mathbf{P} \mathbf{L}+\mathbf{P}$ is finite-dimensional. Finally, it follows from (14), (15), (18) and (19) that $U^{\prime}$ is an ideal of $U(V)$. Note that $U^{\prime}=U^{\circ}(V)$ if char $k \neq 2$.

Now let $z_{i}=\bar{p}\left(x_{i}\right) \in \mathbf{U}_{\pi}(V)$. 
Lemma 3. $U_{n}(V)$ is spanned by the monomials $z_{i_{1}} \cdots z_{i_{i}}$ where $0 \leq r \leq 2 n$.

Proof. Similarly to the proof of Lemma 1 , let $\mathbf{X}_{r}$ be the subspace of $U_{n}(V)$ spanned by the monomials $z_{i_{1}} \cdots z_{i}$, where $0 \leq s \leq r$. If $z_{i_{1}} \cdots z_{i}$, is a monomial with $r>2 n$ then at least one of the $z_{i}$, say $z_{1}$, occurs at least 3 times. Because of (28) we have $z_{i} z_{j} z_{k} \equiv z_{k} z_{j} z_{i} \bmod \mathbf{X}_{1}$. Using this repeatedly, we see that $z_{i} \cdots z_{i}$ is congruent, modulo $X_{r-2}$, to a monomial of the form $\cdots z_{1}^{3} \cdots$ or $\cdots z_{1} z_{i} z_{1} \cdots$. But $z_{1}^{3} \in \mathbf{X}_{1}$ and $z_{1} z_{i} z_{1} \in X_{1}$ by (16). Hence $X_{r}=X_{r-2}$ if $r>2 n$ which shows $\mathbf{U}_{n}(V)=\mathbf{X}_{2 n}$.

Now we finish the proof of the theorem. From the definition of $\mathbf{U}^{\prime}$ it is clear that the map $p: x \mapsto \tilde{p}(x)+U^{\prime}$ is a $\pi$-representation of $V$ in $U(V) / U^{\prime}$, and that $\mathbf{U}(V) / \mathbf{U}^{\prime}$ is generated by 1 and $p(V)$. Hence $\mathbf{U}(V) / \mathbf{U}^{\prime}$ is a homomorphic image of $U_{\pi}(V)$ and therefore finite-dimensional by Lemma 3. Now $U(V)$ is finitedimensional by Lemma 2 .

3. Nilpotence.

3.1. The radical. Let $V$ be a Jordan triple over the ring $k$. The radical of $V$ is

$$
\operatorname{Rad} V=\{x \in V:(x, y) \text { is quasi-invertible, for all } y \in V\} \text {. }
$$

For the basic properties of the radical we refer to [5]. In particular, $\operatorname{Rad} V$ is an ideal of $V$, and if $f: V \rightarrow W$ is a surjective homomorphism of Jordan triples then $\operatorname{Rad} W \supset f(\operatorname{Rad} V)$. A Jordan triple with $\operatorname{Rad} V=0$ is called semisimple. Recall also that an inner ideal of $V$ is a $k$-submodule $I$ such that $P(I) V \subset I$, and an absolute zero divisor is an element $x \in V$ such that $P(x)=0$. It is easily seen that an absolute zero divisor belongs to the radical.

The proof of the following proposition can be found in [5].

Proposition. If $V$ satisfies the descending chain condition on inner ideals then $V$ is semisimple if and only if it contains no absolute zero divisors $\neq 0$.

3.2. Proposition. Let $V$ be a Jordan triple over the ring $k$ satisfying the descending chain condition on inner ideals. Let $R$ be a commutative associative $k$-algebra, let $V_{R}=V \otimes_{k} R$ be the scalar extension of $V$, and define $\varphi: V \rightarrow V_{R}$ by $\varphi(x)=x \otimes 1$. Then $\varphi(\operatorname{Rad} V) \subset \operatorname{Rad} V_{R}$.

Proof. Let $I=\varphi^{-1}\left(\operatorname{Rad} V_{R}\right)$. Since $\varphi$ is a homomorphism of Jordan triples over $k$, this is an ideal of $V$. Let $I_{R}$ be the $R$-submodule of $V_{R}$ generated by $\varphi(I)$. Then $I_{R}$ is an ideal of $V_{R}$, contained in $\operatorname{Rad} V_{R}$. Since tensoring with $R$ is a right exact functor, we have a commutative diagram with exact rows

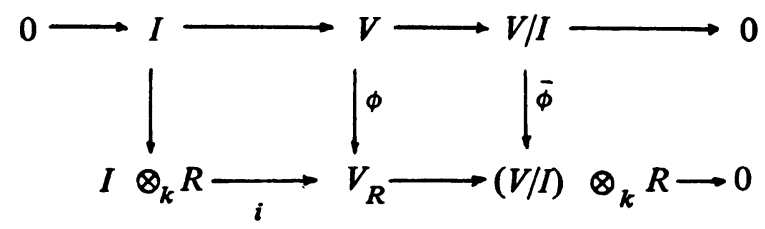


Clearly, $I_{R}=i\left(I \otimes_{k} R\right)$ so that $V_{R} / I_{R}$ may be identified with $(V / I) \otimes_{k} R$. We denote the canonical maps $V \rightarrow V / I$ and $V_{R} \rightarrow V_{R} / I_{R}$ by $x \mapsto \bar{x}$. Assume now that $\bar{x}$ is an absolute zero divisor of $V / I$. Then $\bar{\varphi}(\bar{x})=\overline{\varphi(x)}$ is an absolute zero divisor of $V_{R} / I_{R}$ and thus contained in the radical of $V_{R} / I_{R}$ which is $\left(\operatorname{Rad} V_{R}\right) / I_{R}$. This means $\varphi(x) \in \operatorname{Rad} V_{R}$, i.e., $x \in I$ and therefore $\bar{x}=0$. Since the descending chain condition is inherited by $V / I$ we have $V / I$ semisimple by 3.1 , i.e., $I \supset \operatorname{Rad} V$.

3.3. Theorem. Let $V$ be a finite-dimensional Jordan triple over the field $k$ and assume that $V=\operatorname{Rad} V$. Then $\mathrm{U}^{\circ}(V), \mathrm{U}_{\lambda}^{\circ}(V)$ and $\mathrm{U}_{\sharp}^{\circ}(V)$ are nilpotent.

Proof. By 3.2 and the fact that the universal envelopes are compatible with scalar extensions we may assume $k$ algebraically closed. The crucial fact is

Lemma 1. A finite-dimensional Jordan triple $V$ over an algebraically closed field with $\operatorname{Rad} V \neq 0$ contains an ideal $I \neq 0$ such that $P(I)=L(I, I)=0$.

This is proved in [3]. In case char $k \neq 2$, see also [2].

From now on, we assume $V=\operatorname{Rad} V$ and $k$ algebraically closed.

Lemma 2. $\mathbf{U}_{\lambda}^{\circ}(V)$ is nilpotent.

The proof is by induction on $\operatorname{dim} V$. Let $\bar{l}: V \times V \rightarrow \mathrm{U}_{\lambda}(V)$ be the universal $\lambda$-representation of $V$ in $U_{\lambda}(V)$ (cf. 2.3). If $\operatorname{dim} V=1$, i.e., $V=k . x$, then $P(x) x=0$, and $U_{\lambda}^{\circ}(V)$ is generated by $\bar{l}(x, x)$. By $(26), \bar{l}(x, x)^{4}=0$ and hence $\mathbf{U}_{\lambda}^{\circ}(V)$ is nilpotent. Now let $\operatorname{dim} V>1$. Then by Lemma $1, V$ contains a proper ideal $I$ such that $P(I) V=\{I I V\}=0$. By induction, $U_{\lambda}^{\circ}(I)$ is nilpotent. Let $I$ be the subalgebra of $U_{\lambda}(V)$ generated by $i(I, I)$. Then $I$ is a homomorphic image of $\mathbf{U}_{\lambda}^{\circ}(I)$ and hence is nilpotent. Also, $I$ is contained in the center of $U_{\lambda}(V)$ because of $(25)$ and $\{I I V\}=0$. Therefore I generates a nilpotent ideal $\mathbf{J}=\mathbf{I} \cdot \mathbf{U}_{\lambda}(V)$ of $\mathbf{U}_{\lambda}(V)$.

Let $I^{\prime}$ be the subalgebra of $\mathrm{U}_{\lambda}(V)$ generated by $\bar{l}(I, V)$ and $\bar{l}(V, I)$. Then it follows from (25) and $\{I V I\}=\{I I V\}=0$ that $\mathbf{I}^{\prime} / \mathbf{J} \cap \mathbf{I}^{\prime}$ is commutative. Also by (17), (26), and $P(I)=0$, we have $\bar{l}(x, y)^{4} \equiv 0 \bmod \mathrm{J}$ if $x \in V, y \in I$, or $x \in I$, $y \in V$. By finite-dimensionality, $\mathbf{I}^{\prime} / \mathbf{J} \cap \mathbf{I}^{\prime}$ is nilpotent, and hence $\mathbf{I}^{\prime}$ is nilpotent.

Now let $\mathbf{I}_{\lambda}=\mathbf{I}^{\prime} \cdot \mathbf{U}_{\lambda}(V)$. Then (25) implies

$$
\mathbf{U}_{\lambda}(V) \cdot \mathbf{I}^{\prime} \subset \mathbf{I}_{\lambda}
$$

Hence $I_{\lambda}$ is an ideal of $U_{\lambda}(V)$, in fact, the ideal generated by $\bar{l}(I, V)$ and $\dot{l}(V, I)$. Since $I^{\prime}$ is nilpotent so is $\mathbf{I}_{\lambda}$ by (31). By induction, $U_{\lambda}^{\circ}(V / I)=\mathbf{U}^{\circ}(V) / \mathbf{I}_{\lambda}$ (cf. 2.3) is nilpotent. Hence $U_{\lambda}^{\circ}(V)$ is nilpotent.

We denote by $U^{\prime}$ the ideal of $U(V)$ generated by $\tilde{l}(V, V)$ and $\tilde{p}(V, V)$ (see Lemma 2 in 2.4). 
Lemma 3. $\mathrm{U}^{\prime}$ is nilpotent.

Proof. Let $\mathbf{L}, \mathbf{P}$, and $\mathbf{P}_{+}$be as in the proof of Lemma 2 in 2.4. Then $\mathbf{L}$ is a homomorphic image of $\mathbf{U}_{\lambda}^{\circ}(V)$ and therefore nilpotent by Lemma 2. Also $\mathbf{P}_{+}$is nilpotent since it is contained in $\mathbf{L}$. Let $\mathbf{P}$. be the subspace of $\mathbf{P}$ spanned by the products of an odd number of elements of $\tilde{p}(V, V)$. Then we have $\mathbf{P}=\mathbf{P}_{+}+\mathbf{P}_{-}$, $\mathbf{P}_{-}^{2} \subset \mathbf{P}_{+}, \mathbf{P}_{+} \mathbf{P}_{-}=\mathbf{P}_{-} \mathbf{P}_{+} \subset \mathbf{P}_{-}$, and induction shows $\mathbf{P}^{2 n} \subset \mathbf{P}_{+}^{n}+\mathbf{P}_{+}^{n} \mathbf{P}_{-}$. Hence $\mathbf{P}$ is nilpotent.

From (30) we see that $\mathbf{J}=\mathbf{P L}+\mathbf{P}$ is an ideal of $\mathbf{U}^{\prime}$, and by induction we have $\mathbf{J}^{n} \subset \mathbf{P}^{n} \mathbf{L}+\mathbf{P}^{n}$. Thus $\mathbf{J}$ is nilpotent. Since $\mathbf{U}=\mathbf{L}+\mathbf{J}$ and $\mathbf{U}^{\prime} / \mathbf{J} \cong \mathbf{L} / \mathbf{L} \cap \mathbf{J}$ is nilpotent, $\mathbf{U}^{\prime}$ is nilpotent.

Note. Lemma 2 and Lemma 3 (with a different proof) as well as the concept of $\lambda$-representation are due to C.T. Anderson (cf. [2], §8).

Lemma 4. There exists an ideal $N \neq 0$ of $V$ such that $P(N)=L(V, N)$ $=L(N, V)=0$.

Proof. Let $I$ be the ideal of Lemma 1, and consider the regular representation $\left(L_{I}, P_{I}\right)$ of $V$ in $\operatorname{End}_{k} I$, i.e., $L_{I}(x, y)=L(x, y) \mid I$, and $P_{I}(x)=P(x) \mid I$. Let $f: \mathrm{U}(V) \rightarrow \mathrm{End}_{k} I$ be the induced homomorphism of associative algebras. Then by Lemma $3, f\left(U^{\prime}\right)$ is nilpotent. Thus if $f\left(U^{\prime}\right)^{n} \neq 0$ but $f\left(U^{\prime}\right)^{n+1}=0$ then $N=\left\{x \in I: f\left(\mathbf{U}^{\prime}\right) \cdot x=0\right\} \supset f\left(\mathbf{U}^{\prime}\right)^{n} . I \neq 0$ and $N$ is invariant under $f(\mathbf{U}(V))$ since $U^{\prime}$ is an ideal of $\mathbf{U}(V)$. Hence $N$ is an ideal of $V$ with the desired properties.

Lemma 5. $\mathbf{U}_{\pi}^{\circ}(V)$ is nilpotent.

Proof. We may assume char $k=2$ since otherwise $U_{\sharp}^{\circ}(V)=0$. The proof is by induction on $\operatorname{dim} V$. If $V=0$ there is nothing to prove. Let $V \neq 0$, and let $N$ be as in Lemma 4. From $P(N) V=\{V V N\}=0$, (16) and (28) we obtain

$$
\begin{aligned}
& z x z=0, \\
& x y z=z y x,
\end{aligned}
$$

for $z \in \bar{p}(N)$ and $x, y \in \bar{p}(V)$. We will show that the ideal $\mathbf{N}_{\text {, of }} \mathbf{U}_{\mathbf{z}}(V)$ generated by $\bar{p}(N)$ is nilpotent. Since $\mathbf{U}_{\pi}(V)$ is finite-dimensional, it suffices to show that $\mathbf{N}_{\pi}$ is spanned by nilpotent elements. Now $\mathbf{N}_{\pi}$ is spanned by the monomials $m=x_{1} \cdots x_{r}$ where $x_{i} \in \bar{p}(V)$ and at least one of the $x_{i}$ belongs to $\bar{p}(N)$. Using (33), we see that $m^{3}$ equals a monomial of the form $\cdots z^{3} \cdots$ or $\cdots z x z \cdots$ where $z \in \bar{p}(N)$ and $x \in \bar{p}(V)$. Thus $m^{3}=0$ by (32), and $N_{z}$ is nilpotent. By induction, $U_{\sharp}^{\circ}(V / N)=U_{\sharp}^{\circ}(V) / N_{\S}$ is nilpotent, and the lemma is proved.

Now we finish the proof of the theorem. $\mathbf{U}^{\circ}(V) / \mathbf{U}^{\prime}$ is a homomorphic image of $\mathbf{U}_{\pi}^{\circ}(V)$ and therefore nilpotent by Lemma 5 . Thus $\mathbf{U}^{\circ}(V)$ is nilpotent by Lemma 3. 


\section{BIBLIOGRAPHY}

1. N. Jacobson, Lectures on quadratic Jordan algebras, Tata Institute, Bombay, 1969.

2. O. Loos, Lectures on Jordan triples, University of British Columbia, Vancouver, 1971. 3.—-, On algebraic groups defined by Jordan structures (to appear).

4. K. McCrimmon, Representations of quadratic Jordan algebras, Trans. Amer. Math. Soc. 153 (1971), 279-305. MR 42 \#3139.

5. K. Meyberg, Lectures on triple systems, University of Virginia, Charlottesville, Va., 1972.

6. K. Yamaguti, On representations of Jordan triple systems, Kumamoto J. Sci. Ser. A 5 (1962), 171-184. MR 27 \#3677.

MAthematisches Institut, Universttät MONSTER, 44 MONSTER, West Germany 